\title{
Tumour suppression by Immune System through Stochastic Oscillations
}

\author{
Giulio Caravagna ${ }^{1, *}$, Alberto d'Onofrio ${ }^{2, \dagger}$, Paolo Milazzo ${ }^{1}$ and Roberto Barbuti ${ }^{1}$ \\ ${ }^{1}$ Dipartimento di Informatica, Università di Pisa \\ Largo Pontecorvo 3, 56127 Pisa, Italy. \\ Email: \{caravagn,milazzo,barbuti\}@di.unipi.it \\ ${ }^{2}$ Department of Experimental Oncology, European Institute of Oncology \\ Via Ripamonti 435, 20141 Milano, Italy. \\ Email: alberto.donofrio@ifom-ieo-campus.it
}

\begin{abstract}
The well-known Kirschner-Panetta model for Tumour-Immune System interplay [Kirschner and Panetta, J. Math. Biol 37 (3), 1998] reproduces a number of features of this essential interaction, but it excludes the possibility of tumour suppression by the immune system in the absence of therapy. Here we present a hybrid-stochastic version of that model. In this new framework, we show that in reality the model is also able to reproduce the suppression, through stochastic extinction after the first spike of an oscillation.
\end{abstract}

Keywords: Tumour - Immune System - Immune Surveillance - Immunotherapy Stochastic Model - Hybrid Model

\section{Introduction}

The tumour-immune system interaction takes place because tumour cells are characterised by a vast number of genetic and epigenetic events leading to the appearance of specific antigens, called neoantigens, that trigger antitumoural actions by the immune system (Pardoll 2003). These observations provided a theoretical basis (Burnet 1957) to the empirical hypothesis of immune surveillance, i.e. that the immune system may act to control and also, in some case, to eliminate tumours (Ehrlich 1909). Only in recent years has the study of cancer immunobiology accumulated a sufficient amount of evidence to show that tumours may be suppressed by immune system effectors. This has been made possible by the application of new molecular techniques and by performing a large number of epidemiological studies (Dunn et al. 2004).

The competitive interaction between tumour cells and the immune system involves a considerable number of events and molecules in a extremely complex way. As a consequence, the immune system is not able to eliminate a neoplasm in all cases, since it may escape from its control. Of course, a dynamic equilibrium may also be established, such that the tumour may survive in a microscopic steady state, which is undetectable by diagnostic equipment (d'Onofrio 2007). However, let us consider a tumour which is constrained by the immune system in a microscopic steady state. On the one hand, the presence of a small colony of tumour cells may result in metastases. On the other hand, over a long period of time, a significant fraction of the mean human life span, according to (Dunn et al. 2004), the neoplasm may develop multiple strategies to circumvent the action of the immune system (Whiteside 2002, Pardoll 2003, Dunn et al. 2004, Vicari et al. 2002). This, in the long term,

\footnotetext{
* equal contributor and corresponding author

†equal contributor
} 
may allow it to evade immune surveillance and to re-commence growing to its carrying capacity. The tumour has itself adapted to survive in a hostile environment, in which the antitumour immune response is activated (d'Onofrio 2007, d'Onofrio et al. 2010).

However, the tumour-immune system interaction may also show attractors that are different from a constant equilibrium, namely oscillations. In fact, one may observe both 'short term-small amplitude' oscillations (Kennedy 1970, Vodopick 1972, Gatti et al. 1973, Mehta and Agarwal 1980) and patterns of remission-recurrence such as alternation of long phases of dormancy (where a fallacious apparent immune surveillance seems reached) followed by phases where the tumour seems to escape (Tsao et al. 1997, Blumberg et al. 1990, Sohrabi et al. 1980). Finally, the study of the tumour-immune system interaction led to the proposal and implementation of an interesting therapeutic approach: immunotherapy (De Vito et al. 2005), consisting in stimulating the immune system in order to better fight, and hopefully eradicate, a cancer. The basic idea of immunotherapy is simple and promising, but the results obtained in medical investigations are globally controversial (Agarwala 2003, Bleumer et al 1003, Kaminski et al. 2004).

Regarding the mathematical modelling of the above interactions, many papers have appeared using either a finite dimensional approach (De Lisi and Rescigno 1977, Kirschner and Panetta 1998, Stepanova 1980, Kuznetzov et al. 1994, Kuznetzov and Knott 2001, Galach 2003, Szymanska 2003, De Vladar and Gonzalez 2004, De Pillis et al. 2005, d'Onofrio 2005, d'Onofrio 2007, Cappuccio et al. 2006, Kronik et al. 2008, Kirschner and Tsygvintsev 2009) or the theory of kinetic active particles (Bellomo and Delitala 2008, Bellomo et al 2008).

Some of the above works focus on sustained oscillations that are related to delays in the process of detecting the presence of the tumour by the immune system (Galach 2003, Szymanska 2003, d'Onofrio et al. 2010), whereas others show that oscillations are also possible in the case of absence of such delays (d'Onofrio 2006, Kirschner and Panetta 1998). For example, in (d'Onofrio 2006) an analytical study is conducted and conditions are stated for the existence and uniqueness of a limit cycle for a large class of models.

In (Kirschner and Panetta 1998), Kirschner and Panetta proposed a largely influential tridimensional deterministic model, whose variables are tumour cells, effector cells and the concentration of interleukins. This model is able to explain both the above type of tumour size oscillations, as well as, of course, constant equilibria, both macroscopic and microscopic. The global behaviour of the model was recently investigated in (Kirschner and Tsygvintsev 2009). Although a vast array of behaviours is mimicked by the solutions of the Kirschner-Panetta model, the tumour-free equilibrium is unstable for all biologically meaningful values of the parameters of the model.

However, we noticed that in the transitory oscillations before reaching a stable equilibrium, and also in sustained stable large oscillations, very small values of the tumour size are predicted by this deterministic model. These values could be considered well below the level of detection but they cannot be considered as eradication. Thus, we propose here a stochastic version of the KirschnerPanetta model, which may better describe the dynamics of the tumour-immune system interplay in the case of low levels of both tumour and immune cells. In the bio-mathematical literature it has been stressed how deterministic models may exhibit oscillating solutions having no counterpart in their corresponding stochastic version, where population extinction is, instead, predicted (Nasell 2002).

In order to assess whether this also happens in the Kirschner-Panetta model, we propose here an extended model where the dynamics of tumour cells and immune effectors are described by a suitable stochastic process, whereas the dynamics of the concentration of interleukins is modelled by an ordinary differential equation. Since the stochastic processes are interlinked with the dynamics of the interleukins (and vice versa), the main features of this model are that it contains time-dependent propensity functions and combines both the features of deterministic and stochastic models.

This provides a useful tool through which we aim to offer a theoretical contribution towards a better understanding of the dynamical aspects of a key phenomenon: the suppression of a tumour by the immune system. In particular, we focused on: $i$ ) assessing the role of antigenicity of tumour cells (Kirschner and Panetta 1998) in shaping those dynamics; ii) investigating the interplay between the so-called intrinsic noise and the macroscopic tendency of tumour-immune system interplay to oscillate (d'Onofrio 2006, Kirschner and Panetta 1998). 
The present work is organised as follows: in Section 2 we present the Kirschner-Panetta model and we summarise its main features, with particular regard to those linked to the onset of sustained oscillations. In Section 3 we propose a hybrid stochastic version of the model. In Section 4.1 we use the stochastic Kirschner-Panetta model to investigate the role of stochasticity in tumour suppression. The work ends with some concluding remarks.

\section{Overview of the Kirschner-Panetta model}

In (Kirschner and Panetta 1998) the following model of the dynamics of tumour-immune system interaction was proposed:

$$
\begin{aligned}
T_{*}^{\prime} & =r T_{*}\left(1-b T_{*}\right)-\frac{a T_{*}}{g_{2}+T_{*}} E_{*} \\
E_{*}^{\prime} & =\frac{p_{1} I}{g_{1}+I} E_{*}-\mu_{2} E_{*}+c T_{*}+s_{1} \\
I^{\prime} & =\frac{p_{2} T_{*} E_{*}}{g_{3}+T_{*}}-\mu_{3} I+s_{2}
\end{aligned}
$$

where $T_{*}(t), E_{*}(t)$ and $I(t)$ denote, respectively, the densities of tumour cells, effectors of the immune system and interleukins. The tumour induces the recruitment of the effectors at a linear rate $c T$, thus, the parameter $c$ may be seen as a measure of the immunogenicity of the tumour, i.e. 'a measure of how different the tumour is from self' (Kirschner and Panetta 1998). The proliferation of effectors is stimulated by the interleukins. A continuous infusion immunotherapy is delivered; in the general case, both effectors and interleukins are injected at rates $s_{1} \geq 0$ and $s_{2} \geq 0$. The source of interleukin is modelled as linearly depending on effectors, and it also depends on the tumour burden. Finally, the average lifespan of effectors is $\mu_{2}^{-1}$ and the average degradation time for interleukin is $\mu_{3}^{-1}$.

In the absence of therapy $\left(s_{1}=s_{2}=0\right)$, the main results obtained in (Kirschner and Panetta 1998) are the following: the unique tumour suppression equilibrium point $(0,0,0)$ is always unstable, there is a value $0<c_{0}<<1$ such that for $0<c<c_{0}$ there are three equilibria of which two are unstable and one is locally stable. As it is intuitive, the antigenicity of the tumour being very small, this locally stable equilibrium is very close to the carrying capacity in the absence of immune response. Furthermore, there is a value $c_{\max }>0$ such that if $c \in\left(c_{0}, c_{\max }\right)$ there is a unique periodic solution; the period and amplitude of the limit cycle decreases as far as $c$ approaches $c_{\max }$. Finally, when $c>c_{\max }$ there is a unique globally stable equilibrium, which is reached through damped oscillations. Again, this equilibrium is a decreasing function of $c$, and its size is small due to the high immunogenicity of the tumour. The presence of a very small equilibrium state that is not detectable might seem to be a sub-optimal outcome, so that one may consider it as practically equivalent to tumour suppression. However, it must be remembered that, as we mentioned in the introduction, the Volterrian interaction between tumour cells and the immune system is also evolutionary: tumour cells evolve their ability to evade the control (Dunn et al. 2004, d'Onofrio 2007) and, as a consequence, no microscopic steady state may be considered permanent and safe for the patient (d'Onofrio 2007).

Thus, we note that this model explicitly precludes the possibility of tumour suppression, which follows from the absence of a baseline influx of effectors. We remark that this holds in the absence of therapy, and that is the case which we will consider in the rest of the paper. In the presence of therapies, the behaviour of the system becomes more complex, but in all the possible combinations of the parameters it is possible to find regions where globally stable limit cycles exist, as well as regions where there is cancer suppression. Moreover, there is a threshold value such that, for the injection rate of the interleukins greater than such a value, there is an unbounded growth of effectors, leading to capillary leak syndrome. 


\section{A Hybrid Stochastic version of the Kirschner-Panetta model}

In this section we define a hybrid stochastic version of the model (1). In principle, indeed, before defining a stochastic equivalent of the model (1), we should switch from variables representing densities to variables representing the total number of cells. Thus, we would obtain an ODE model from which it would be straightforward to obtain a three dimensional stochastic process. However, since the molecular weight of interleukins is 15000 Dalton, the average number of proteins is huge. As a consequence, we may assume that the dynamics of $I(t)$ is well approximated by a linear differential equation with randomly varying coefficients, which, however, in the intervals between two consecutive stochastic events are evidently constant.

As a consequence, we introduce as a new parameter the volume $V$ of interest (e.g. the blood and bone marrow volumes for leukaemia) and we define:

$$
T_{*}=\frac{T}{V}, E_{*}=\frac{E}{V}
$$

thus obtaining:

$$
\begin{aligned}
T^{\prime} & =r T\left(1-\frac{b}{V} T\right)-\frac{a T}{g_{2} V+T} E \\
E^{\prime} & =\frac{p_{1} I}{g_{1}+I} E-\mu_{2} E+c T+V s_{1} \\
I^{\prime} & =\frac{p_{2}}{V} \frac{T E}{g_{3} V+T}-\mu_{3} I+s_{2}
\end{aligned}
$$

From the above model, we define a hybrid stochastic process, where a bi-dimensional stochastic process is linked to a scalar differential equation ruling the dynamics of the concentration of interleukins. Namely, in the interval between the $\mathrm{n}$-th event (at time denoted as $\left.t_{n}\right)$ and the $(n+1)$-th event, the above mentioned ODE is:

$$
I^{\prime}=\frac{p_{2}}{V} \frac{T\left(t_{n}\right) E\left(t_{n}\right)}{g_{3} V+T\left(t_{n}\right)}+s_{2}-\mu_{3} I
$$

which, since in $\left(t_{n}, t_{n+1}\right)$ the other two state variables are constant, is a linear ordinary differential equation with constant input and constant coefficients. Thus, the solution of (4) in $\left(t_{n}, t_{n+1}\right)$ has the following analytical form:

$$
I(t)=B_{n}+\left(I_{n}-B_{n}\right) \operatorname{Exp}\left(-\mu_{3}\left(t-t_{n}\right)\right)
$$

where $B_{n}$, for the sake of notation simplicity, is the following function of $\left(T_{n}, E_{n}\right)$ :

$$
B_{n}:=\frac{1}{\mu_{3}}\left(\frac{p_{2}}{V} \frac{T\left(t_{n}\right) E\left(t_{n}\right)}{g_{3} V+T\left(t_{n}\right)}+s_{2}\right) .
$$

The stochastic model of the evolution of the tumour cells and the effector cells is defined by the following events and the corresponding propensity functions:

$$
\begin{array}{ll}
\left(R_{1}\right) & T \mapsto T+1 \\
\left(R_{2}\right) & T \mapsto T-1 \\
\left(R_{3}\right) & T \mapsto T-1 \\
\left(R_{4}\right) & E \mapsto E+1 \\
\left(R_{5}\right) & E \mapsto E-1 \\
\left(R_{6}\right) & E \mapsto E+1 \\
\left(R_{7}\right) & E \mapsto E+1
\end{array}
$$

$$
\begin{array}{r}
a_{1}=r_{2} T \\
a_{2}=r_{2} b V^{-1} T^{2} \\
a_{3}=(a T E) /\left(g_{2} V+T\right) \\
a_{4}(t)=p_{1} E(I(t) /(g 1+I(t))) \\
a_{5}=\mu_{2} E \\
a_{6}=c T \\
a_{7}=V s_{1}
\end{array}
$$

Notice that all the propensity functions except for $a_{4}(t)$ are time-constant. In order to analyse this model, we need to define an algorithm for computing its time evolution. Although in the literature 
algorithms for simulating hybrid systems have been presented (Salis and Kaznessis 2005, Alfonsi et al. 2004), in this model we combine the hybrid approach with time-dependent propensity functions (Lecca 2006, Anderson 2007). Consequently, we define, based on the main ideas of Gillespie's algorithm (Gillespie 1976, Gillespie 1977), a stochastic evolution algorithm for this hybrid model.

The event-induced changes in the values of $T$ and $E$ can be represented by the following sevendimensional vectors

$$
\nu_{T}=(1,-1,-1,0,0,0,0)^{T r}
$$

and

$$
\nu_{E}=(0,0,0,1,-1,1,1)^{T r} .
$$

Namely, $\nu_{T}[i]$ and $\nu_{E}[i]$ denote how the event $R_{i}$ affects the population $T$ and $E$, respectively. Furthermore, we denote with $S$ the set of time-constant propensity functions, namely the set $\left\{a_{1}, a_{2}, a_{3}, a_{5}, a_{6}, a_{7}\right\}$.

The unique time varying propensity is given by the formula:

$$
a_{4}(t)=E_{n} \frac{p_{1} I(t)}{g_{1}+I(t)}
$$

We define the function:

$$
A(\tau)=\int_{t_{n}}^{t_{n}+\tau} a_{4}(t) d t
$$

Given the analytical form of $I(t)$ in formula $(5), A(\tau)$ reads as follows:

$$
A(\tau)=\frac{p_{2} E_{n}}{\left(B_{n}+g_{1}\right) \mu_{3}}\left(\tau \mu_{3}\left(B_{n}+g_{1}\right)+g_{1} \operatorname{Ln}\left(\frac{g_{1}+I_{n}}{-B_{n}+e^{\tau \mu_{3}}\left(B_{n}+g_{1}\right)+I_{n}}\right)\right) .
$$

The exact simulation algorithm for the hybrid system under study can consequently be defined as follows:

1. Input the initial state: $\left(T_{0}, E_{0}, I_{0}\right)$ and the maximum simulation time $\Theta_{M}$

2. Initialise the time $t=t_{0}$ and the system state $(T, E, I)=\left(T_{0}, E_{0}, I_{0}\right)$.

3. If $t \geq \Theta_{M}$ end the simulation, else

4. Evaluate all the $a_{j}$ with $j \in S$ and their sum $a_{0}=\sum_{j \in S} a_{j}$.

5. The putative time for the next reaction $\tau$ is determined by solving the following transcendental equation:

$$
A(\tau)+a_{0} \tau=\chi
$$

where $\chi$ is a random number with distribution $\operatorname{Exp}(1)$. For the numerical solution of this equation we used Newton-Raphson method.

6. Given a random number $r$ uniformly distributed in the interval $[0,1]$, the next event is determined in accordance with

$$
\sum_{i=1}^{j-1} a_{i}<r \cdot a_{0} \leq \sum_{i=1}^{j} a_{i}
$$

7. Update the system state to $\left(T+\nu_{T}[j], E+\nu_{E}[j], I(t+\tau)\right)$ and the clock to $t+\tau$.

8. Go to step 3.

Finally, in order to perform the simulations of both the deterministic and stochastic model, we have to define numerical values for the parameters.

Concerning the parameters, we used the values and ranges given in the references (Kirschner and Panetta 1998, Kirschner et al 2004), as listed in Table 1. Note that those values pertain to mice, since they were taken from the influential papers (DeBoer et al. 1985, Kuznetzov et al. 1994), where accurate fitting of real data concerning laboratory animals were performed. 


\begin{tabular}{|c|c|c|c|}
\hline Parameter & Value & Measure Unit & Description \\
\hline$r_{2}$ & 0.18 & days & baseline growth rate of the tumour \\
\hline$b$ & $10^{-9}$ & $m l^{-1}$ & carrying capacity of the tumour \\
\hline$a$ & 1 & $m l \times d a y s^{-1}$ & baseline strength of the killing rate by immune effectors \\
\hline$g_{2}$ & $10^{5}$ & $m l^{-1}$ & $50 \%$ reduction factor of the killing rate by immune effectors \\
\hline$p_{1}$ & 0.1245 & $m l \times d a y s^{-1}$ & baseline strength of the interleukin-stimulated growth rate of effectors \\
\hline$g_{1}$ & $2 \times 10^{7}$ & $m l^{-1}$ & $50 \%$ reduction factor of interleukin-stimulated growth rate of effectors \\
\hline$\mu_{2}$ & 0.03 & $d a y s^{-1}$ & inverse of average lifespan of effectors \\
\hline$c$ & - & $t i m e^{-1}$ & tumour antigenicity \\
\hline$p_{2}$ & 5 & $m l \times d a y s^{-1}$ & baseline strength of production rate of interleukins \\
\hline$g_{3}$ & $10^{3}$ & $m l^{-1}$ & loss/degradation rate of $I L_{2}$ \\
\hline$\mu_{3}$ & 10 & $t i m e^{-1}$ & blood and bone marrow volumes for leukaemia \\
\hline$V$ & 3.2 & $m l$ & injection rate of effectors for adoptive cellular immunotherapy \\
\hline$s_{1}$ & 0.0 & $m l^{-1} \times d a y s^{-1}$ & injection rate of interleukins for immunotherapy \\
\hline$s_{2}$ & 0.0 & $m l^{-1} \times d a y s^{-1}$ &
\end{tabular}

Table 1: Parameters used in most of the simulations of the model. Tumour antigenicity $c$ is the only variable parameter that is reported in the description of each simulation.

As far as parameter $V$ is concerned, by taking into account that in a chimeric mouse the body weight ranges from 20 grams for female mouse up to $40 \mathrm{grams}$, and that their blood volume ranges from $5.8 \mathrm{ml}$ per $100 \mathrm{grams}$ up to $8 \mathrm{ml}$ per $100 \mathrm{grams}$, it follows that a reasonable range for $V$ is from $1.16 \mathrm{ml}$ up to $3.2 \mathrm{ml}$, in our simulations we assumed: $V=3.2 \mathrm{ml}$.

In our simulations we focused on two key parameters: $c$, which reflects the antigenic potential of the tumour, and $s_{1}$ which represents the injection rate of effectors for adoptive cellular immunotherapy.

\section{Stochastic behaviour}

\subsection{Stochastic suppression of tumour in absence of immunotherapy}

In (Kirschner and Panetta 1998) it has been shown that the antigenicity parameter $c$ is a key parameter for model (1) since, for very low values of $c$, the tumour burden reaches values near its carrying capacity, for large values of $c$ the tumour reaches a small steady state, whereas, for intermediate values, lying in a quite narrow range $\mathcal{R}=\left(c_{\min }, c_{\max }\right)$, there is the onset of oscillations through Hopf bifurcations at $c=c_{\min }$ and at $c=c_{\max }$. This is biologically sound since, of course, the immunogenicity of a tumour is an essential factor so that the immune system may attack the tumour with some effectiveness.

However, simulating (3) we noticed that, although for $c$ slightly higher than $c_{m i n}$ the maximum value attained during the oscillations may be of the order of the carrying capacity, the minima of $T(t)$ seem very small. Indeed, if we plot time courses of tumour size in a $\log 10$ scale, as in Figure 1 , we may easily see that there are temporal ranges where the deterministic setting predicts that $T(t)<1$ cell.

This suggested to us that a range $\Omega \subset \mathcal{R}$ might exist where, by using the stochastic version of the Kirschner-Panetta model, the oscillating interplay tumour-effectors-interleukins might lead to the eradication of the disease.

This hypothesis was confirmed by the stochastic simulations. All the simulations of the hybrid model weredone by using a Java implementation of this model and of the algorithm presented in Section 3. The Java source code of the implementation is freely available at the URL: http://www . di . unipi.it/msvbio.

We present, in Figure 2, two realisations (for $c=0.01$ and for $c=0.02$ ) of the stochastic process corresponding to the deterministic time-courses shown in Figure 1. We set the worst possible initial conditions for effectors and interleukins, i.e. $E(0)=0, I(0)=0$.

It is immediately noticeable that the stochastic behaviour is deeply different from that predicted by the deterministic model since, in the stochastic simulation, only the first spike of the oscillation is achieved and then the tumour eradication is reached. In particular, with $c=0.01$ eradication is observed around day 160 and with $c=0.02$ around day 120 . 

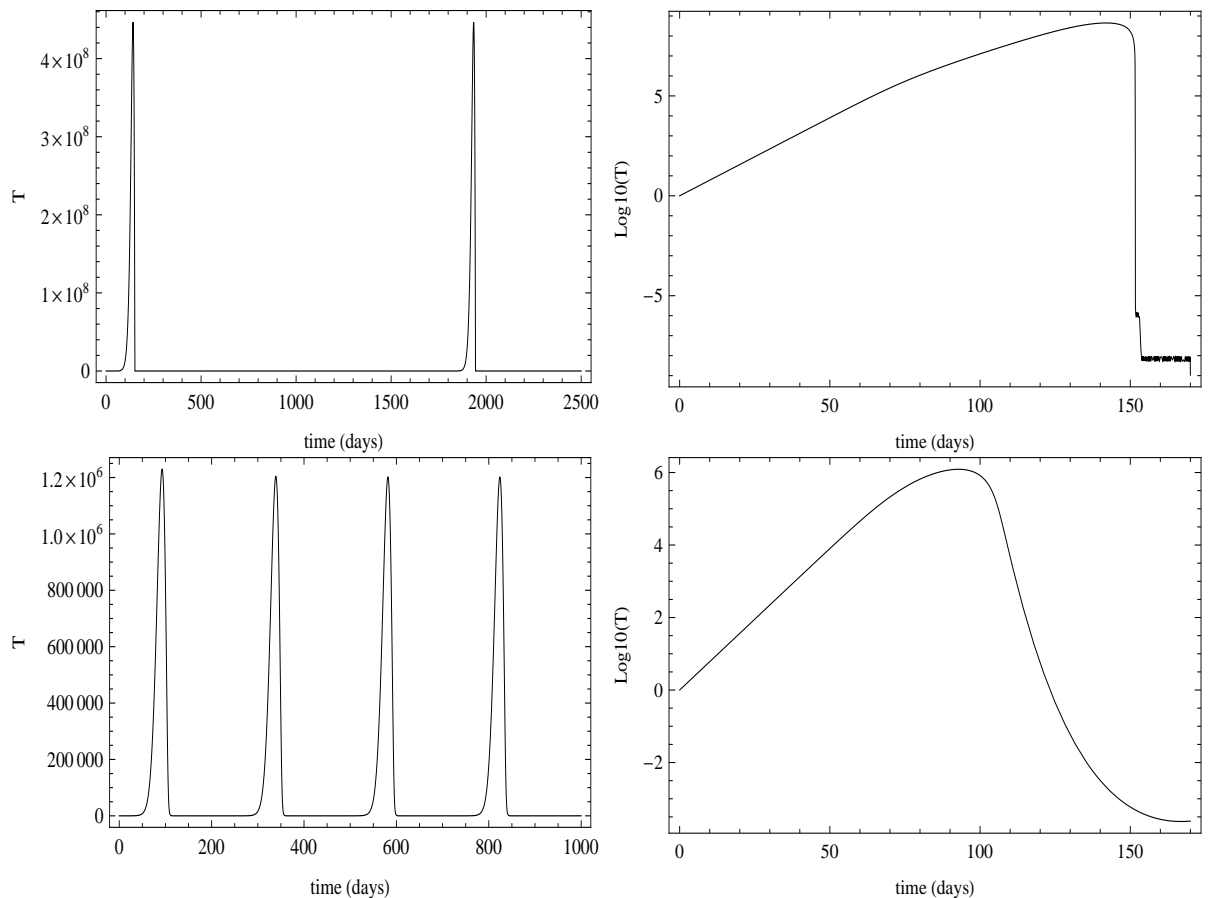

Figure 1: Deterministic dynamics of $T(t)$ for $c=0.01$ (upper panels), and $c=0.02$ (lower panels). In left panels the curves are plotted in natural scale, whereas in right panels the plot is in $\log 10$ scale. The other parameter are as in Table 1.

Thus, denoting as $t_{e r}$ the random variable time of occurrence of the eradication we calculated the associated empirical probability density $\varrho\left(t_{e r} ; c\right)$ with $c=0.02$. The result is shown in Figure 3. Such a density, computed over $10^{3}$ stochastic runs of the model, has a peak at around day 130; all the simulations have shown eradication with a simulation time bounded to 200 days.

Note that the stochastic simulations for some values of $c$ become computationally intractable for sample hundreds of runs. For example, at $c=0.01$ a single stochastic run took almost 70 hours on a Apple MAC ${ }^{1}$.

Consequently, we decided to perform a deeper analysis on the deterministic model, which is much less computationally expensive, considering as eradication the first time instant in which $T(t)<1$. We calculated, for the values of $\log _{10}(c)$ in $[-4 ;-1]$ the average eradication time $\left\langle t_{e r}\right\rangle(c)$ in function of $c$, as reported in Figure 4. We note that for $c$ approaching $c_{m i n}$ the putative eradication time becomes very large. However, notice that in reality the patient dies before that the carrying capacity is reached. Moreover, after decreasing for nearly all the range $\Omega$, the curve has a minimum and the eradication time restarts growing. Notice that for $c=0.02\left(\sim \log _{10}(c)=-2.6\right)$ the analysis of the deterministic model gives roughly similar predictions of the stochastic behaviour observed in Figure 3 .

In order to assess the influence of non-null initial value for effectors, in Figure 5, we show the effect of increasing $E_{0}$ on the empirical probability density of $t_{e r}$ with $c=0.02$ in the stochastic model. These densities are computed over $10^{2}$ stochastic runs. All the initial configurations we considered are of the form $\left(T_{0}, E_{0}, I_{0}\right)=\left(1,10^{i}, 0\right)$ with $i=1, \ldots, 5$. In particular, for $i=1,2,3$ the densities have the peaks in the interval $[120 ; 140]$ while, for $i=4,5$ the densities become bimodal with peaks in the intervals $[120 ; 140]$ and $[0 ; 10]$. For the particular initial configurations, which are not shown in the figure, having $10^{6}$ or more initial effectors, all the eradications are observed almost instantaneously.

The same kind of analysis was performed by varying the initial numbers of interleukins. In particular, in Figure 6 , we show the effect of increasing $I_{0}$ on the empirical probability density of $t_{e r}$ with

\footnotetext{
${ }^{1}$ Apple Macbook 2.4 Ghz Intel Core 2 Duo, 4GB 1067 Mhz running Mac OS X 10.5.8.
} 

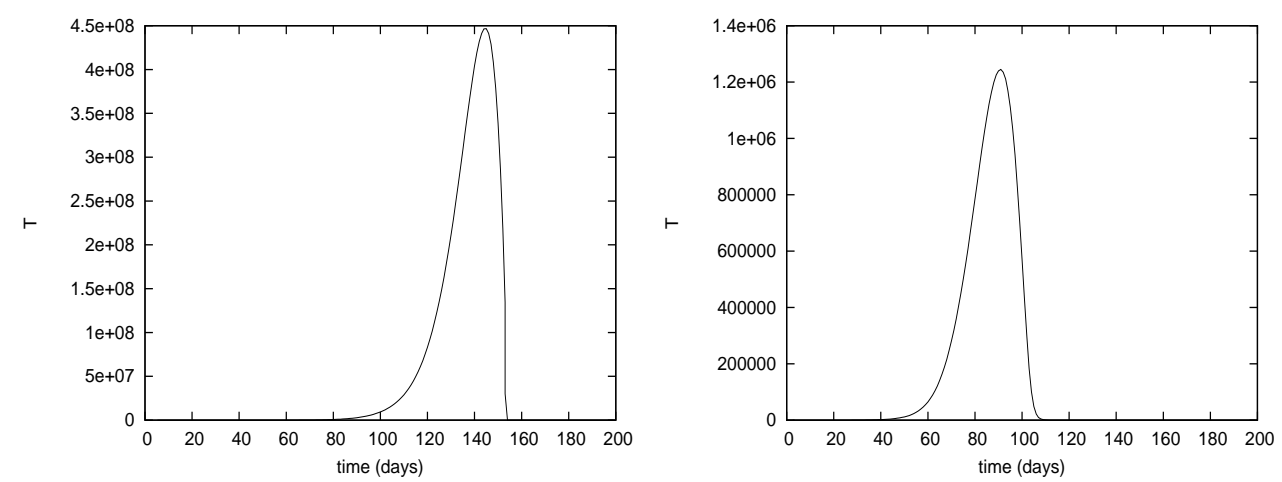

Figure 2: Stochastic dynamics of $T(t)$ for $c=0.01$ (left panel), and $c=0.02$ (right panel). The other parameter are as in Table 1. In both cases there is tumour suppression, and further spikes of the original oscillations disappear.

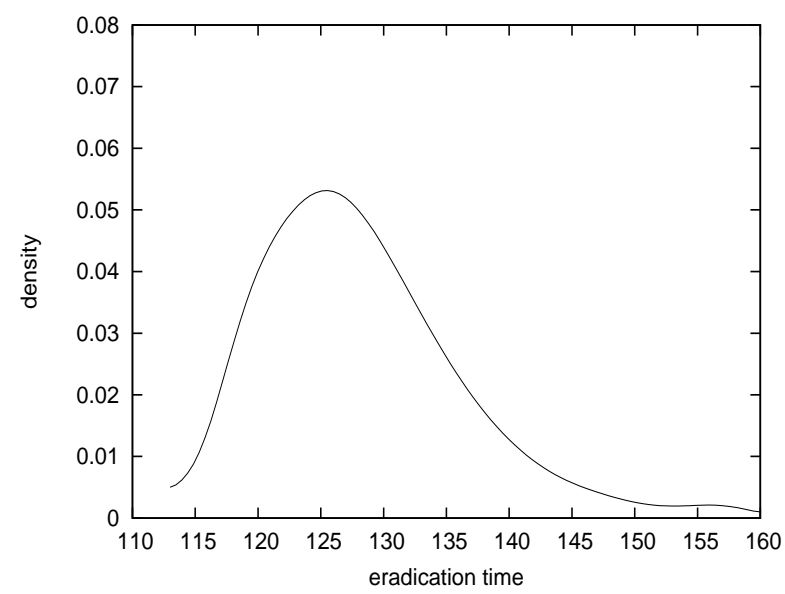

Figure 3: Empirical probability density of $t_{e r}$ with $c=0.02$ computed over $10^{3}$ stochastic runs. The other parameters are as in Table 1.

$c=0.02$ in the stochastic model. Also these densities are computed over $10^{2}$ stochastic runs. All the initial configurations we considered are of the form $\left(T_{0}, E_{0}, I_{0}\right)=\left(1,0,10^{i}\right)$ with $i=1,2,4,6$. In this case, unlike the previous ones, we observed single modal probability densities with peaks in the interval $[110 ; 135]$.

\subsection{Stochastic oscillations in absence of immunotherapy}

The stochastic version of the Kirschner-Panetta model exhibits stochastic oscillations for a small range of values of the antigenicity

$$
0.03 \approx<c<0.032
$$

whereas for higher values damped oscillations appears (i.e. with $c=0.033$ the system reaches an equilibrium with around 60000 tumour cells). In Figure 7 we show two examples of oscillations; for $c=0.03$ and for $c=0.032$. Note that in Figure 7 we also plotted the minimum after the first spike of the oscillation. 

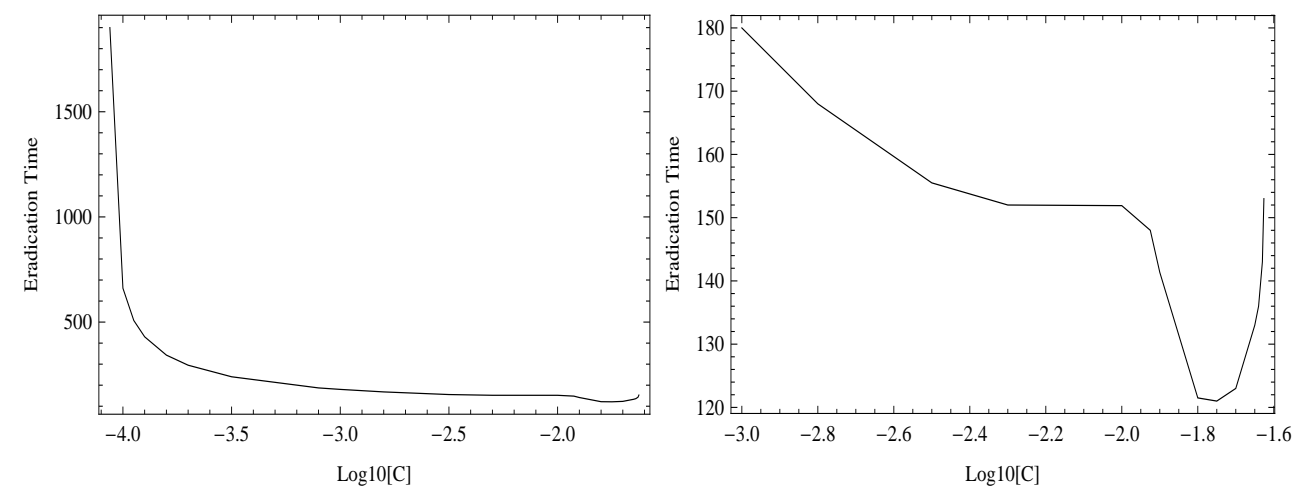

Figure 4: Eradication time vs. $\log _{10}(c)$ for the deterministic model. The other parameters are as in Table 1. Left panel: complete plot, right panel: zoom to final part.
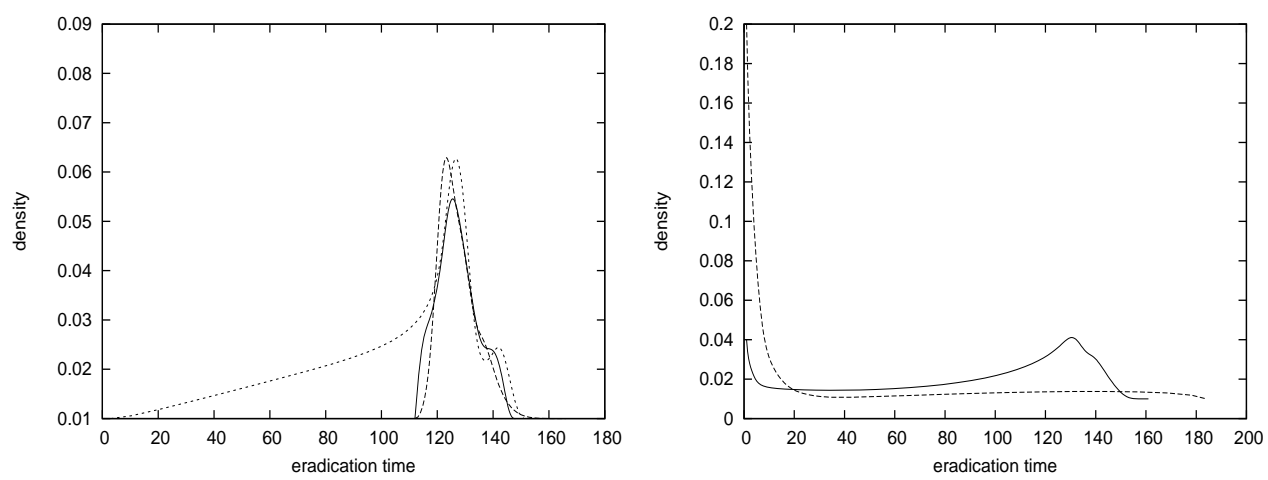

Figure 5: Empirical probability density of $t_{e r}$ with $c=0.02$ and variable initial size of effectors: $E(0)=10^{i}$ with $i=1$ (solid line), $i=2$ (dashed line), $i=3$ (semi-dashed line) on the left and $i=4$ (solid line) and $i=5$ (dashed line) on the right. For each configuration the density has been computed over $10^{2}$ stochastic runs. The other parameters are as in Table 1.

\subsection{Tumour suppression in the case of large $c$}

If the value of $c$ is further increased the average value of the stochastic oscillations of tumour cells progressively reduces. The reduction is such that the suppression of the tumour gradually reappears. For example, we performed a series of simulations by varying $c$ (2000 simulations 2000 days long for each value of $c$ ), and we observed that at least one observed eradication occurred for $c$ greater than a threshold value 13 , the value for which two eradications over 2000 simulations of the stochastic process were observed. At $c=30$ tumour eradication was observed in $20 \%$ of simulations, and for $c=50$ almost half of the simulations had the tumour eradication as outcome (956 out of 2000).

In Figure 8 one simulation of the stochastic process for $c=71.1$ is shown, whereas in Figure 9 (left) we plotted the percentage of observed eradications in function of $c$. As far as the times when the first eradication is observed, we note that in many cases, the minimal eradication time is very small. Thus, we plotted in Figure 9 (right) the modal value of $t_{\text {erad }}$ for $c \in(13,71.5)$, i.e. the value of $t_{e r}$ for which the maximum number of eradications was observed.

\subsection{Immunotherapy}

Although, as we stressed in the introduction, here we are essentially interested in investigating the effect of stochasticity in the unperturbed time course of a tumour, for the sake of completeness, we briefly report some simulations of an adoptive cellular immunotherapy $\left(s_{1}>0\right)$. 


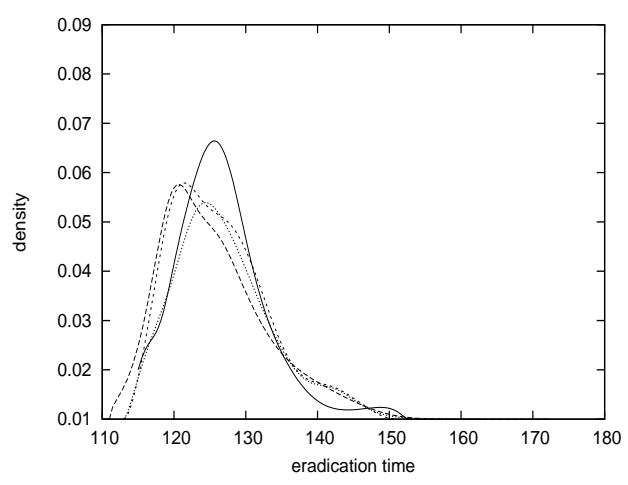

Figure 6: Empirical probability density of $t_{e r}$ with $c=0.02$ and variable initial size of interleukins: $I L(0)=10^{i}$ with $i=1$ (solid line), $i=2$ (dashed line), $i=4$ (semi-dashed line) and $i=6$ (dotted line). For each configuration the density has been computed over $10^{2}$ stochastic runs. The other parameter are as in Table 1.
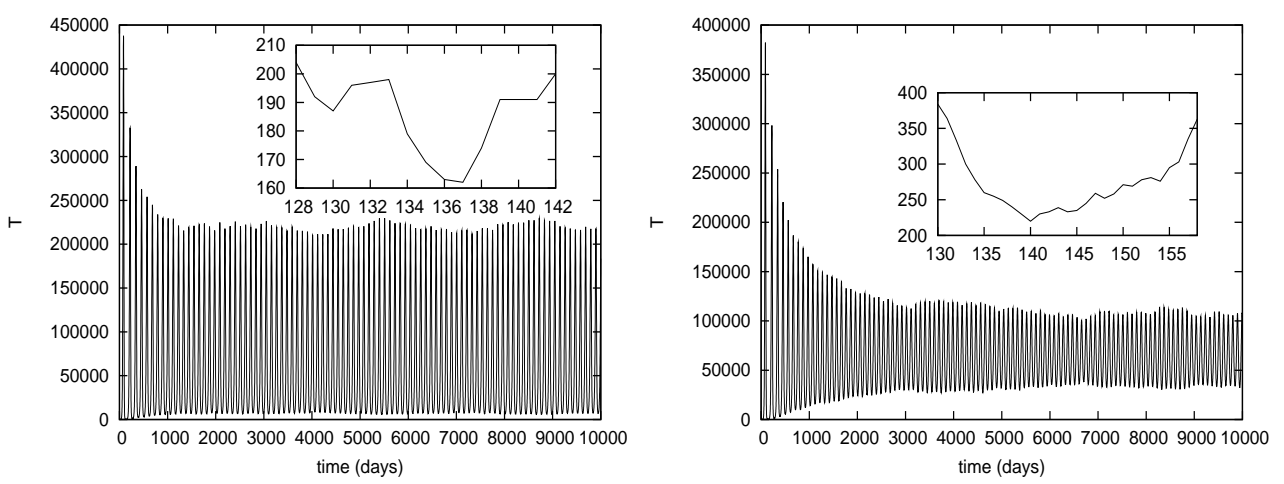

Figure 7: Stochastic oscillations for $c=0.03$ (left) and $c=0.032$ (right). The other parameters are as in Table 1. In both subfigures an insert is plotted zooming in on the minimal values reached at the end of the first spike of the oscillation.

The deterministic setting is quite pessimistic since a therapy only eradicates for $t \rightarrow \infty$, so that after a therapy having a realistic finite time span the tumour restarts growing. Thus the maximum beneficial effect of an immunotherapy would be moving the state of tumour from the region of convergence of a macroscopic equilibrium (not compatible with the life of the host organism) to the basin of attraction of a small steady state.

The stochastic simulations, instead, suggests that an immunotherapy may eradicate the disease in a finite time, as shown, for the case of an adoptive cellular immunotherapy (Figure 10).

We focused on the antigenicity value $c=10^{-4}$ which is, for both the deterministic and stochastic models, a value such that no eradication is observed in the absence of therapy. Regarding the therapy, we modelled an adoptive cellular immunotherapy from day 70 to day 130 of simulation. We used $s_{1}=0.0$ for the first 70 days of simulation and, for the following days, we used $s_{1}=10 s 1_{c r}$ where $s 1_{c r}$ is the threshold value for the local stability of the suppression in the deterministic setting (Kirschner and Panetta 1998).

\section{Concluding Remarks}

In order to investigate the phenomenon of spontaneous onco-suppression by the immune system and at the same time to assess whether the Kirschener-Panetta approach may reproduce this important 


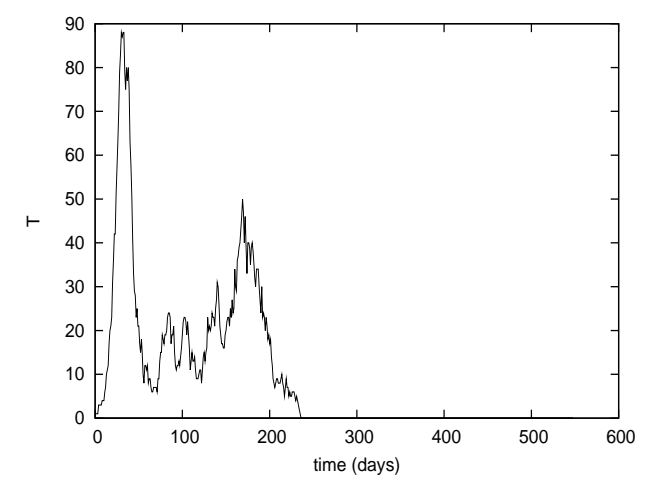

Figure 8: Stochastic suppression for $c=1.1 c^{*}$. The other parameters are as in Table 1.
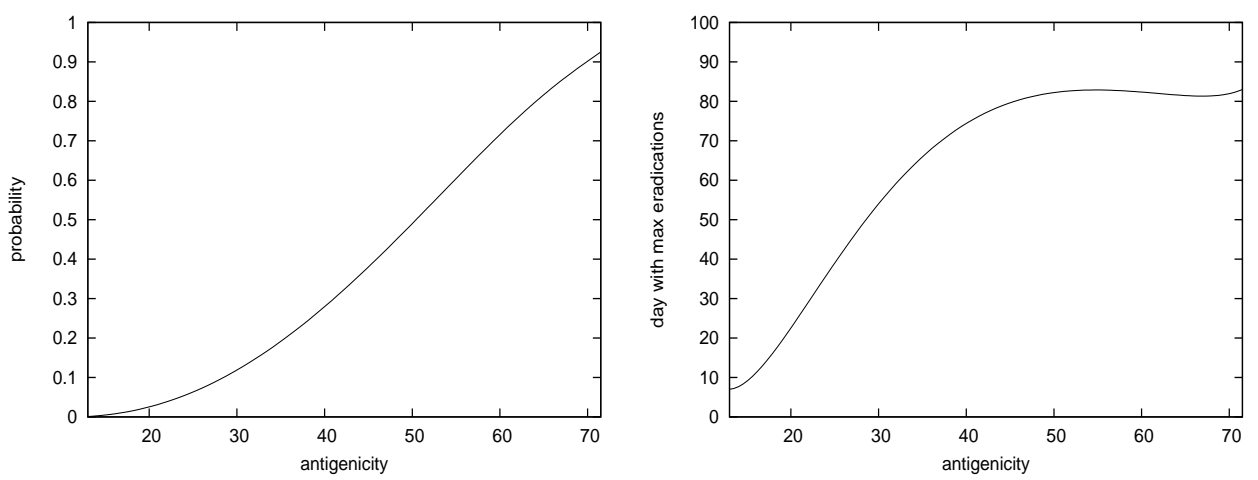

Figure 9: In the left panel, the percentage of eradication obtained in function of the parameter $c$. In the right panel, the modal value of $t_{\text {erad }}$ in function of the antigenicity parameter $c$ for $c \in(13,71.5)$. The other parameters are as in Table 1.

phenomenon, we proposed here a hybrid model that extends the Kirschner-Panetta model. In our extended model the dynamics of tumour cells and immune effectors are described by a suitable stochastic process, whereas the dynamics of the concentration of interleukins are modelled by an ordinary differential equation. Since the stochastic processes are interlinked with the dynamics of the interleukins (and vice versa), the main features of this model are that it contains time-dependent propensity functions and combines both the features of deterministic and stochastic models.

In order to simulate the model, it was necessary to define a proper stochastic simulation algorithm. Indeed, although in the literature we find algorithms for simulating time-dependent propensity functions (Lecca 2006, Anderson 2007) as well as algorithms for simulating hybrid models (Salis and Kaznessis 2005, Alfonsi et al. 2004), the combination of both is missing. Consequently, for the sake of analysing this model we defined an ad-hoc algorithm based on the ideas of Gillespie's Stochastic Simulation Algorithm (Gillespie 1976, Gillespie 1977) which permits an efficient implementation.

We would point out that the model we present is not a completely new one, but is a stochastic model built on top of a well-known and biologically backgrounded model. The simulations and their biological interpretation, instead, are new.

Our hybrid stochastic version of the Kirschner-Panetta model suggests that, at least in some cases, complete immune surveillance with suppression of the neoplasm might be reached thanks to the conjunction of the intrinsic tendency of the tumour-immune system to oscillate, which is significantly evidenced by the deterministic model, with the stochastic dynamics. We note that this may empirically be inferred by the deterministic Kirschner-Panetta model by considering the tumour eradicated whenever $T(t)<1$. In fact, from a quantitative analysis of the models, we observe significant differences in the tumour eradication times. Moreover, by following the stochastic approach we have 

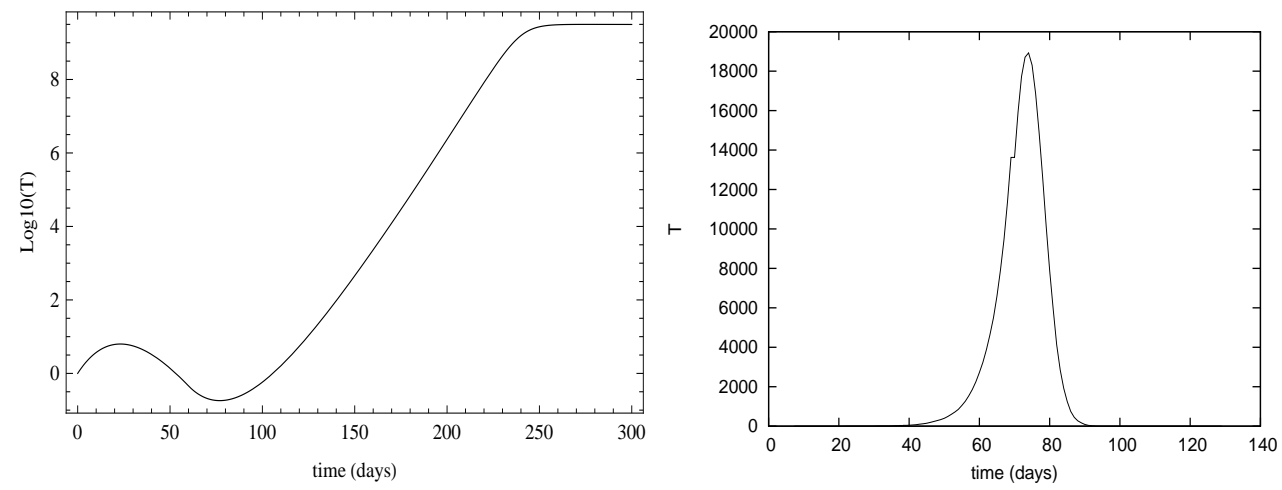

Figure 10: Deterministic vs stochastic models of adoptive cellular immunotherapy with $c=10^{-4}$ and $s_{1}=5400$ delivered for 60 days after day 70. The other parameter are as in Table 1.

been able to give a probability distribution of the eradication time, and the discussion of Section 4.1 regarding the different behaviours of the system by varying its initial conditions would not have been possible by considering only the average eradication time provided by the deterministic model. The relevant role of oscillations showed an intriguing role for the immunogenicity of the disease, which is represented by the parameter $c$ in the model. Indeed, the deterministic immunogenicity path can be summarised as follows: for low values of $c$ the tumour size is large and the oscillations have very large periods, for intermediate values of $c$ we observe intermediate oscillating tumour size. Finally, for large values of $c$ we observe small tumour size reached after damped oscillations.

Conversely,, the stochastic path forecasts immune suppression for intermediate $c$, as well as, of course, for quite large $c$. This is quite a surprising result which deserves further biological investigations and which may have interesting implications for some immunotherapies, since for intermediate $c$ the suppression range is small.

Moreover, since for many cases of tumour suppression in the case of low $c$ tumour sizes do reach very large values, we must stress that in these cases the eradication is only theoretical since the host unfortunately dies. However, for larger values of $c$ the tumour size is large but compatible with life, so that the model may contribute to a better understanding some sporadic cases of spontaneous permanent regression of macroscopic tumours (Krikorian 1980). Of course for large values of $c$ the probability of having a remission is large (e.g. for $c=50$ we observed that the suppression is reached in $50 \%$ of the simulated paths). Finally, we observe that also stochastic sustained oscillations are possible.

When considering adoptive cellular immunotherapies, our simulations suggest that a stochastic model may qualitatively be used in tuning the average length of the therapies, as briefly illustrated in Figure 10.

In summary, our work shows that the Kirschner and Panetta model is even more powerful than it seems, since our stochastic version of it may also describe the most important phenomenon of the tumour-immune system interplay, namely the total suppression of the tumour by the immune system in the absence of human intervention.

Finally, as far as the phenomena of late recurrence of tumours are concerned, since they did not appear in our stochastic simulations, we think that they may have their main root in the phenomenon of immunoediting (Dunn et al. 2004, d'Onofrio 2007) and we intend to study them, following the approach used in (d'Onofrio 2007), by allowing, in the stochastic model, a slow decrease of some key parameters.

\section{Acknowledgements}

The work of A. d'Onofrio was conducted within the framework of the EU Integrated Project "Advancing Clinico-Genomic Trials on Cancer - ACGT". The ACGT project is partly funded by the EU 
and the authors are grateful for this support. This work was conducted within the framework of the official agreement on "Computational Medicine" between the European Institute of Oncology and the University of Pisa. We wish to thank the anonymous referees for their suggestions that contributed to significantly improving the paper.

\section{References}

Agarwala, S.A., 2003. New Applications of Cancer Immunotherapy, S. A. Agarwala (Guest Editor), Seminars in Oncology, Special Issue 29-3 Suppl. 7.

Alfonsi, A., Cances, E., Turinici, G., Di Ventura, B., Huisiga, W., 2004. Exact simulation of hybrid stochastic and deterministic models for biochemical systems. INRIA Tech. Report 5435.

Anderson, D.F., 2007. A modified next reaction method for simulating chemical systems with time dependent propensities and delays J. Chem. Phys. 127, 214107.

Bleumer, I., Oosterwijk, E., de Mulder, P., Mulders, P.F., 2003. Immunotherapy for Renal Cell Carcinoma. European Urology $44,65-75$,

Bellomo, N., and Delitala, M. 2008. From the mathematical kinetic, and stochastic game theory to modelling mutations, onset, progression and immune competition of cancer cells. Phys. of Life Rev. 5, 183-206.

Bellomo, N., Li, N.K., Maini, P.K. 2008 On the foundations of cancer modelling: Selected topics, speculations, and perspectives. Math. Mod. and Meth. in Appl. Sc. 18, 593-646.

Blumberg, N., Chuang-Stein, C., Heal, J. M., 1990. The relationship of blook transfustion, tumor staging, and cancer recurrence. Transfusion, 30(4), 291-294.

Burnet, F.M., 1957. Cancer - a biological approach. Brit. Med. J. 1, 841-847.

Cappuccio, A., Elishmereni, M., and Agur Z. 2006. Cancer immunotherapy by interleukin-21 treatment strategies evaluated in a mathematical model. Canc. Res. 66, 7293-7300.

Cattani, C., Ciancio, A., d'Onofrio, A. 2010. Metamodeling the learning-hiding competition between tumours and the immune system: A kinematic approach. Math. and Comp. Mod. In Press: doi:10.1016/j.mcm.2010.01.012.

DeBoer R.J, Hogeweg P., Hub F., Dullens J., DeWeger R.A. and DenOtter W. 1985. Macrophage T Lymphocyte interactions in the anti-tumor immune response: A mathematical model. J. Immunol. 134, 2748-2758.

d'Onofrio, A., Gatti, F., Cerrai, P., 2010. Delay-induced oscillatory dynamics of tumour-immune system interaction. Math. and Comp. Mod. 51, 572-591.

d'Onofrio, A., 2007. Tumour evasion from immune system control: strategies of a MISS to become a MASS. Chaos solitons and Fractal 31, 261-268.

d'Onofrio, A., 2005. A general framework for modeling tumour-immune system competition and immunotherapy: Mathematical analysis and biomedical inferences. Phys. D 208 , 220-235.

d'Onofrio, A., 2006. Tumour-immune system interaction: modeling the tumour-stimulated proliferation of effectors and immunotherapy. Mathematical Models and Methods in Applied Sciences 16, 1375-1401.

Dunn, G.P., Old, L.J., Schreiber, R.D., 2004. The three ES of Cancer Immunoediting. Ann. Rev. of Immun. 22, 322-360. 
Ehrlich, P., 1909. Ueber den jetzigen Stand der Karzinomforschung. Ned. Tijdschr. Geneeskd. 5, 273-290.

Galach, M., 2003. Dynamics of the tumour-immune system competition: the effect of time delay. Int. J. Appl. Math Comp. Sc. 13, 395-406.

Gatti, R., et al., 1973. Cyclic Leukocytosis in Chronic Myelogenous Leukemia: New Perspectives on Pathogenesis and Therapy . Blood 41, 771-783.

Gillespie, D., 1977. Exact Stochastic Simulation of Coupled Chemical Reactions. J. of Phys. Chem. 81, 2340-2361.

Gillespie, D., 1976. A General Method for Numerically Simulating the Stochastic Time Evolution of Coupled Chemical Reactions. J. of Comp. Phys. 22 (4), 403-434.

Holzman, M., Carlton, C.E.Jr., Scardino, P.T., 1991. The frequency and morbidity of local tumor recurrence after denitive radiotherapy for stage c prostate cancer. The J. of Ur. 146, 1578-1582.

Lecca, P., 2006. A time-dependent extension of Gillespie algorithm for biochemical stochastic $\pi$ calculus. Proc. of the 2006 ACM symposium on Applied computing, 137-144.

Kaminski, J.M., Summers, J.B., Ward, M.B., Huber, M.R., Minev, B., 2004. Immunotherapy and prostate cancer. Canc. Treat. Rev. 29, 199-209.

Kennedy, B.J., 1970. Cyclic leukocyte oscillations in chronic myelogenous leukemia during hydroxyurea therapy. Blood 35, 751-760.

Kirschner, D., Panetta, J.C., 1998. Modeling immunotherapy of the tumour - immune interaction. J. Math. Biol. 37, 235-252.

Kirschner, D., Arciero, J. C. and Jackson, T. L., 2004. A Mathematical Model of Tumor-Immune Evasion and siRNA Treatment. Discr. and Cont. Dyn. Systems 4, 39-58.

Kirschner, D., and Tsygvintsev, A. 2009. On the global dynamics of a model for tumor immunotherapy. Math. Biosc. and Eng. 6(3), 573-583.

Krikorian, J.G., 1980. Spontaneous regression of non/hodgkin lymphoma: a report of nine cases. Cancer 46, 2093-2099.

Kronik,N., Kogan, Y., Vainstein,V., and Agur Z. 2008. Improving alloreactive CTL immunotherapy for malignant gliomas using a simulation model of their interactive dynamics. Cancer Immunol Immunother 57, 425-439.

Kuznetsov, K.A., Knott, G.D., 2001. Modeling tumour regrowth and immunotherapy, Math. Comp. Mod. 33, 1275-1287.

Kuznetsov, V.A., Makalkin, I.A., Taylor, M.A., Perelson, A.S., 1994. Nonlinear dynamics of immunogenic tumours: Parameter estimation and global bifurcation analysis. Bull. Math. Biol. 56, 295-321.

De Lisi, C., Rescigno, A., 1977. Immune surveillance and neoplasia: a minimal mathematical model. Bull. Math. Biol. 39(2), 201-221.

Mehta, B.C., Agarwal, M.B., 1980. Cyclic oscillations in leukocyte count in chronic myeloid leukemia. Acta Hematologica 63, 68-70.

Nani, F., Freedman, H.I., 2000. A mathematical model of cancer treatment by immunotherapy. Math. Biosc. 163, 159-199.

Nasell, I., 2002. Measles outbreaks are not chaotic, in Mathematical approaches for Emerging and Reemerging Infectious Diseases: Models, Methods, and Theory, The IMA Volumes in Mathematics and its Applications, Vol. 126, C. Carlos-Castillo (ed.) Springer- Verlag, New York, 85-114. 
Pardoll, D., 2003. Does the Immune System See Tumours as Foreign or Self?.Ann. Rev. of Immun. 21, 807-839.

De Pillis, L.G., Radunskaya, A.E., Wiseman, C.L., 2005. A Validated Mathematical Model of CellMediated Immune Response to Tumour Growth. Cancer Res. 65, 7950-7958.

Salis, H., Kaznessis, Y., 2005. Accurate hybrid stochastic simulation of a system of coupled chemical or biochemical reactions. J. Chem. Phys. 122, 054103.

Sohrabi, A., Sandoz, J., Spratt, J.S., Polk, H.C., 1980. Recurrence of breast cancer: Obesity, tumor size, and axillary lymph node metastases. JAMA 244(3), 264-265.

Stepanova, N.V., 1980. Course of the immune reaction during the development of a malignant tumour. Bioph. 24, 917-923.

Szymanska, S., 2003. Analysis of the immunotherapy models in the context of cancer dynamics. Int. J. Appl. Math Comp. Sc. 13, 407-418.

Tsao, H., Cosimi, A.B., Sober, A.J., 1997. Ultra-late recurrence (15 years or longer) of cutaneous melanoma. Cancer 79(12), 2361-2370.

Vicari, A.P., Caux, G., Trinchieri, G., 2002. Tumour escape from immune surveillance through dendritic cell inactivation. Sem. Canc. Biol 12, 33-42.

De Vito Jr. V. T., Hellman, J., Rosenberg, S.A., 2005. Cancer: principles and practice of Oncology, J. P. Lippincott.

De Vladar, H.P., Gonzalez, J.A., 2004. Dynamic response of cancer under the influence of immunological activity and therapy. J. Th. Biol. 227, 335-348.

Vodopick, H., Rupp, E.M., Edwards, C.L., Goswitz, F.A., Beauchamp, J.J., 1972. Spontaneous cyclic leukocytosis and thrombocytosis in chronic granulocytic leukemia. New Engl. J. of Med. 286, 284290.

Whiteside, T.L., 2002. Tumour-induced death of immune cells: its mechanisms and consequences. Sem. Canc. Biol. 12, 43-50. 